\title{
Dimostrazione delle condizioni caratteristiche perchè una curva sia di diramazione di un piano quadruplo.
}

\author{
Memoria di Oscar Chisini (a Milano).
}

Sunto. - Le relasioni funzionali, relative a nodi e euspidi, ovviamente necessarie affinchè ma eurva piana $\varphi_{\mathrm{m}}$ sia di diramaztone per un piano quadruplo proveniente dalla proiezione di una superficie d'ordine $4+v$ dotata di un punto $v$-plo, vengono qui dimo. strate essere sufficienti, e ciò in modo semplice e per qualunque valore di $v$.

Si completa tale risultato con la dimostrazione che tutti i piani multipli diramati da una $\varphi_{\mathrm{m}}$ del tipo indicato sono birazionalmente identici.

\section{Introduzione.}

La effettiva caratterizzazione delle curve di diramazione dei piani multipli comineia col lavoro di B. SEGRE (") dedicato ai piani $n$-pli relativi a superfici generali dell' ordine $n$ : base ne è il Teorema di HALPHEN che permette di riconoscere quando una curva gobba $d$ 'ordine $m \cdot n$ è intersezione completa di due superfici degli ordini $m$ ed $n$. Seguono i lavori di ChIsINr e Mavara $\left({ }^{2}\right)$ sui piani tripli nel cosi detto caso semplice (superfici d' ordine $3+v$ dotate di un punto $\gamma$-plo e del resto generali) e in un ulteriore caso poco più ampio.

Indirizzo diverso hanno i lavori della signora Masontr-BIggiogero $\left({ }^{3}\right)$ i quali tendono a caratterizzare le curve di diramazione come inviluppi di convenienti sistemi di curve piane plurikangenti, collegandosi così ad una fondamentale osservazione di O. CHIstri (1) e a successivi lavori di G. PoMPIL (5). Nei suoi lavori la MasotT si preoceupa essenzialmente di mostrare le possibilità del metodo, saggianłone le difficoltà e superandole nei primi casi. Essa, dopo aver ripreso i piani tripli nel caso semplice, tratta $i$ primi due esempi di piani quadrupli (sempre del caso semplice).

(1) B. Segre, Sulla caratterizzazione delle curve di diramazione dei piani multipli ge. nerali, "Mem. Aco. ditalia", Vol. Io, N. 4, (1930), pp. 1.31.

(2) O. ChISIN e C. F. MANARA, Sulla caratterizzazione delle curve di divamazione dei piani tripli, "Annali di Mat. s, Serie LV, Tomo XXV (1946), pp. 255.266 e "Boll. U.M.I.". Serie III, anno III, (1948), pp. 6-8.

(3) G. Masotti Biggrogero, La caratterizzazione della curva di diranazione dei piani tripli ottenuta mediante sistemi di curve plwritangenti, "Renr. Istit. Lombnrdos, Vol. 80, (1947), pp. 151.160 ; Sulla caratterizzazione della curva di diramazione dei piani quadrupli generali, a Rend. Isti . Lombardo", Vol, 81, (1947), pp. 269.280; Sulla caratterizzazione delle curve di diramazione dei piani quadrupli, "Rend. Istit. Lombardo", Vol. 81, (1948).

(4) O. ChIsINI, Sulla identitò birazionale delle funzioni algebriche di due variabili dotate di wna medesina curva di diranazione, "Rend. Istit. Lombardo ", Fol, 77, (1943-44), pp. 339-356.

(5) G. Pompils, Su una classe di piani multipli vigati, "Rend, di Mat. e delle sue annlieazioni s Serie $V$ Vol 5 (1946) nn $57-74$ 
Ora qui dimostro in generale la condizione perchè una eurva $\varphi$ sia di dirumazione per un piano quadruplo del caso semplice (superficie d'ordine $4 i v$ dotata di punto $v-p l o \mid$ e cio riconducendomi a dimostmre che la y proiezione da un punto $O$ della intersezione (completa) di una superficie di ordine $4+v$ dotata di $O y$-plo e della poline di $O$ rispetto ad essa: tale via, più diretta, si accosta, por questo particolare, alla trattazione di B. SEgre. Penso che la dimostrazione si possa estendere ai piani $n$-pli, sempre del easo semplice.

Il risultato da dimostrare è il seguente

Teorema. - Una curva piana $\varphi_{m}$ è di diramazione per un piano quadruplo del tipo semplice, relativo ad una superficie di ordine $4+v$ quando il suo ordine $m$ ed $\mathrm{i}$ numeri $\delta$ e $k$ dei suoi nodi $\theta$ delle sue cuspidi soddisfino le relazioni aritmetiche

$$
\left\{\begin{array}{l}
m=6 v+12 \\
\delta=4(v+1)(v+3) \\
k=(v+2) m
\end{array}\right.
$$

ed inoltre siano soddisfatte le seguenti relazioni funzionali di equivalenza

$$
\left\{\begin{array}{l}
K \equiv(v+2) R \\
N^{\prime}+4 T \equiv 2(v+1) R \\
R+T \equiv Z
\end{array}\right.
$$

dove $K$ indica il gruppo delle cuspidi, $N^{\prime}$ il gruppo $N$ dei nodi considerati cia. scano su entrambi i rami di $\varphi_{m}$ che vi passano, $R$ un grupo di punti di $\varphi_{m}$ allineati, $T$ un gruppo di punti non virtuale, $Z$ un gruppo di punti tutti di. versi dai punti $T$.

si noti che la seconda delle (2) implica che il gruppo $N^{\prime}$ sia contenuto nella serie $2(v+1) R$ e la terza delle (2) porta che la serie $|R+T|$ abbia dimensione almeno 3 e sia sprovvista di punti fissi.

Il teorema ora enunciato, anche se non espresso in questa forma, deve considerarsi appartenere alla signora MASOTTI, in quanto la generalizzazione dei casi da lui indicati al caso generale è del tutto ovria. Qui, per semplicita, effettuo la dimostrazione nel caso, ehe può considerarsi caratteristico,

$$
v=5, \quad m= \pm 2, \quad \delta=192, \quad k=294 ;
$$

mettendo ai gruppi che interessano un indice che ne fissi l'ordine si ha:

$$
R_{4 z}, K_{294}, N_{19 z}, N_{344}, T_{30} .
$$

Si potranno tralasciare questi indici quando essi non giovino.

Indicheremo con una sola lettera tanto nna eurvi quanto il polinomio primo membro della equazione; il fattore di proporzionalita di questo polinomio sarà scelto in modo da semplificare lo formule, senza bisogno di ovvie 
ad intili precisazioni. Le eurve indicate con $\psi$ si intendono sempre essere aggiunte alla curva $\varphi$.

Infine, a coronamento della trattazione, faremo vedere che tatti i piani maltipli (non importa fissare che siano quadrupli) diramati da una $\varphi$ del tipo indicato sono tra loro birazionalmente identici.

\section{Preliminari.}

Come abbiamo detto vogliamo dimostrare il

Thorkma. - Una curva pinna $\varphi_{12}$ è li diramazione per un piano quadruplo (proiezione di una superficie $F_{9}$ del nono ordine da un suo punto quintaplo, e del resto generale) quando abbia un gruppo $N_{192}$ di 192 nodi e un gruppo $K_{294}$ di 294 cuspidi ed esista un gruppo $T_{30}$ di 30 punti tali che sia

$$
\left\{\begin{array}{l}
K_{294} \equiv 7 R_{42} \\
N_{384}^{\prime}+4 T_{30}=12 R_{42} \\
R_{43}+T_{30} \equiv Z_{72}
\end{array}\right.
$$

dove, ripetiamo, $N^{\prime}$ è il gruppo dei nodi considorati, ciascuno, su entrambi i rami di $\varphi$ che lo contengono $\theta Z$ non ha punti in comune con $T$.

Osservaziona. - Come di consuetudine in queste questioni la $\varphi_{42}$ è in. tesa irriducibile e generica entro la famiglia (cui appartiene) delle curve con uguali caratteri jlückeriani.

Per giungere alla dimostrazione del teorema, in questo paragrafo esponiamo anzitutto, con qualche variante di dimostranione, alcane proposizioni preliminari dovute anch' esse alla Signora Masoter $\left({ }^{6}\right)$.

Lempa 1. - Esiste una curva $p_{26}$ (d'ordine 26) che passa per i. nodi e le cuspidi di $\hat{\varphi}_{1:}$ e tocea questa. con intersezione quadripunta, nei 30 punti del gruppo $T_{30}$.

Infatti: la $\varphi_{4,}$ ha il genere 334 ; sia $G_{606}$ un suo grtuppo canonico segato da una (particolare) aggiunta $\psi_{39}$. Il gruppo

$$
2 K+N^{\prime}+4 T
$$

per le prime due relazioni (3) ¿ segato (fuori di $K_{284}$ ed $N_{192}$ ) da una curva aggiunta d' ordine $39+26$ passante ulteriormente per il delto $G_{666}$. Questa aggiunta dovrà passare doppiamente per le cuspidi e per i punti doppi $(K$ ed $N /$ cioè essere una biaggiunta. Poichè il $G_{666}$ appartiene ad una aggiunta $\bigcup_{30}$, segue che il grappo $4 T$ apparterra ad una agginnta d'ordine 26 (e la indichiamo con $p_{26}$ ) come afferma l'enunciato.

Lwma 2. - La serie complota $|R+T|$ o segata dalle aggiunte $\psi_{27}$ aventi (con la $\varphi_{42}$ ) contatto tripunto nei punti di $T$.

Ciò ov ovio; inoltro, per le ipotesi ammesse, la detta serie ha almono la dimensione 3 e le $\psi_{27}$ suddette non hanno contat to maguiore (al tripunto) fisso in alcun punto $T$.

(b) Cfr. la teran delle note sopra citate. 
Lemua 3. - Le suddette $\psi_{a r}$ segano lia $p_{: 5}$ (oltre che nei punti $N$ e $K$ ) in un gruppo $\Gamma_{126}$ che è di contatto (con la $p_{26}$ ) per una curva $\vartheta_{12}$ tangente anche nel gruppo $T$.

Si osservi infatti che su $\varphi_{42}$ tatti i gruppi equivalenti a $2 R+2 T$ sono segati dalle aggiunte $\psi_{28}$ passanti per $2 T$; quindi, considerata una $\psi_{27}$ (per $3 T)$ e il gruppo $G_{7:}$ che essa sega su $\varphi_{42}$, esiste una $\psi_{28}$ cho sega tale gruppo contato due volte, onde risulta che si può serivere:

$$
\psi_{27}^{2}=\vartheta_{12} \varphi_{43}+p_{26} \psi_{28}
$$

dove, ovviamente, la $\vartheta_{12}$ soddisfa alle condizioni enunciate.

\section{Relazioni essenziali.}

Veniamo ora a stabilire le proposizioni essenziali che conducono al risul. tato prelisso.

Lemma 4. - Le $\psi_{34}$ per $3 T$, ohe segano su $\varphi_{42}$ la serie $|4 R+T|$ tagliano la $p_{26}$ foltre che in $\left.3 T\right)$ nel gruppo fisso $\Gamma_{126}$ e pertanto si scrivono nella forma

$$
\psi_{30}=x_{3} a_{27}+x_{4} p_{26}
$$

dove $a_{27}$ indica una particolare (ma generica) $\psi_{27}$ passante per $3 T$ mentre $x_{3}$ ed $x_{4}$ indicano due generici polinomi in $x$ e $y$ d'urdine uguale al loro indice.

Osserviano infatti che sulla $\eta_{28}$ le $\psi_{30}(p e r 3 T)$ segano la serie $\Gamma_{126}+3 R_{26}^{\prime}$, dove $R_{26}^{\prime}$ indica un gruppo ai 26 punti di $p_{26}$ allineati. Questa serie (speciale) si ottiene ampliando la serie $3 R_{26}^{\prime}$ con l'addizione del gruppo $\Gamma_{126}$ il quale, e occorre dimostrarlo, rimane fisso. Per dimostrare questo fatto basta (per il cosi detto lemma di riduzione) riconoscere che il $\Gamma_{126}$ impone proprio 126 condizioni alle curve $\tau_{3 y}$, d'ordine 23 , che segano su $p_{26}$ la serie canonica, già passanti per un gruppo $3 R_{26}^{\prime}$, cioè alle curve $\tau_{26}$ d' ordine 20 . A tale scopo si osservi che il $\Gamma_{126}$ appartiene ad una $\xi_{10}$ di gensre $p=55$. Sn questa le $\tau_{20}$ segano una serie ovviamente non speciale (d'ordine 240 ) di dimensione $r=240-55$; similmente le $\tau_{20}$ passanti per il $\Gamma_{126}$ segano una serie d'ordine 114 e didimen. sione $s=114-55$, aneora non speciale. Segue $r-s=126$, e quindi il fatto che i 126 punti $a \Gamma_{126}$ impongono 126 condizioni alle $\tau_{20}$ e quindi che la dimensione della serie $\left|\Gamma_{4: 6}+3 R_{36}^{\prime}\right|$ ò uguale a quolla della serie $\left|3 R_{26}^{\prime}\right|$ vale a dire che le $\psi_{30}$ per il $3 T$ passano tutte per il $\Gamma_{126}$.

Osservazrone. - Il ragionamento d̀ valido anche se la $p_{26}$ o la $9_{13}$ non fossero generiche ma presentassero delle complicanioni, ad esempio un punto del $\Gamma_{126}$ fosse doppio per la $p_{26}$ (e quindi nnoho per la $\left.\vartheta_{12}\right)$; in tali casi sono ovvie le varianti da apportarsi al ragionmento.

Teorema 5. - La anrie $|R+T|$ bua $y_{\text {ig. }}^{3}$.

La dimostrazione è un facile corollario del Lemma precentente. Notiamo che la serie suddetta è tagliata dal sistema delle $\psi_{27}$ yer $3 T$. Sia $\bar{\psi}_{27}$ una di esse. Si consideri la $\psi_{30}$ formata da questa $\psi_{2}$ e dia una generica cubica $\gamma_{3}$. 
Si ha allora

$$
\bar{\psi}_{27} \gamma_{3}=x_{3} a_{27}+x_{4} p_{26}
$$

il che 'nostra che la $\bar{\psi}_{7}$ passa per i punti comuni a $a_{2 \gamma}$ e $p_{26}$ e quindi si serive

$$
\bar{\psi}_{27}=a_{27}+x_{1} p_{26} \quad\left(x_{1} \text { polinomio del prim'ordine }\right) ;
$$

questo esprime essere $\infty^{3}$ il sistema delle $\psi_{2 \gamma}$ (per $3 T$ ) che segano la serie $|R+T|$ cioè appunto essere 3 la dimensione di questa.

Osservazione. - Notiamo che la nostra curva $\varphi_{42}$ si può considerare proiezione, dal punto improprio dell'asse $z$, di una curva gobba $\varphi_{\gamma 2}^{*}$ sulla quale i piani segano la serie (che si proietta nella) $|R+T|$; questa, $\varphi_{72}^{*}$ è data dalla rappresentazione monoidale:

$$
z=\frac{a_{27}}{p_{26}} \text { con } \varphi_{12}=0 .
$$

I punti $T$ della $\varphi_{i 2}$ sono le proiezioni del punto $Z_{\aleph}$, improprio dell'asse $\varepsilon$, considerato su ciascuno dei 30 rami di $\varphi_{72}^{*}$ che vi passano. Portiamo ora la nostra attenzione a questa curva gobba $\varphi_{72}^{72}$ e alle serie segate su di questa. Dobbiamo stabilire due teoremi essenziali, di cui il primo è il

Teonema 6. - Entro il sistema delle superfici $F_{3}$ del nono ordine dotate del punto $Z_{\infty}$ come quintuplo, superfici date dalle equazioni

$$
F_{9}=x_{5} z^{1}+x_{6} z^{3}+x_{7} z^{2}+x_{8} z+x_{9}=0,
$$

ne esistono almeno 5 linearmente indipendenti le quali tutte contengono la $\varphi_{72}^{*}$.

Qui, come innanzi, ciascuna $x_{s}$ indica un polinomio - variabile d'ordine $s$ nelle coordinate $x$ e $y$.

Osserviamo intanto che queste superfici $F$, staccano 5 volte il punto $Z_{\infty}$ su cirscuno dei rami di $\varphi_{72}^{*}$ che vi passano e pertanto staccano, sulla $\varphi_{72}^{*}$, gruppi della serie $S=\left|9 R^{2}+4 T\right|$.

Ci importa valutare la dimensione $r$ di questa serie, e a tal fine ne determiniamo l'indice li specialità, $i$, sulla base del teorema di Rremans-Roch.

La serie canonica $C$ è la serie $|13 R+4 T|$, quindi la serie residua è la serie

$$
|C-S|=|4 R|
$$

la quale è segata (su $\varphi_{42}$ ) dalle $\psi_{30}$ per $3 T$ cui si imponga un ulteriore passaggio per $T$. Ora ogni $\Psi_{30}$ per $3 T$ si serive nella forma

$$
x_{3} a_{27}+x_{4} p_{26}
$$

e quindi - ammesso per il momento che nessuna cubica passi per i 30 punti del $T$ - le $\psi_{30}$ per $4 T$ si scrivono nella forma

$$
x_{4} p_{26}
$$

e la serie $|4 R|$ risulta avere la dimensione 14 , cioè otteniamo

$$
i=15 \text {. }
$$


Se invece per il $T_{3 \prime \prime}$ passano le cubiche $\gamma_{3}$ di un sistema lineare $\infty^{s-1}$ ' allora lo $\psi_{3,}$ per $4 T$ si scrivono

e l'indice di specialita risulta

$$
\begin{gathered}
Y_{3} t_{27}+x_{4} p_{90} \\
i=13 \cdot \mathrm{H} .
\end{gathered}
$$

In definitiva si ha che la timensione dellit serie $\mid 9 R+4 T$ e

$$
r=\frac{19}{49}-334+15+s=179+s
$$

dove $s=0$ quando mancassero le suddette cubiche $\gamma_{3}$.

D'altra parte le superfici $F_{9}$ formano un sistema di dimensione 184 (se ne contano subito i coeficienti). Nessuna di esse, che non contenga la $\varphi_{72}^{*}$, puo tagliare su questa lo stesso gruppo stacento da una supurficie $\gamma_{3} x, z^{5}=0$ we pare folto ancora il punte $Z_{w}$ contato 5 volte su ciaseuno dei rami della $\left.\varphi_{72}^{*}\right)$ appartiene alla serie $|9 R+4 T|$.

Infatti, qualora la superficie

$$
F_{9}=a_{5} z^{4}+a_{6} z^{3}+a_{7} z^{2}+a_{8} z+a_{9}=0
$$

tagliasse su $\varphi_{\tau_{2}}^{*}$ lo stesso gruppo che la $\Phi_{9}=\theta_{3} x, z^{5}=0$ che contiene, contato 5 volte, il gruppo staccato dal piano $z=0$, questo dovrebbe appartenere ai coni $a_{m}=0(m=9 \ldots 5)$; e poichè $z=0$ b un generico piano dello spazio, la $\vartheta_{72}^{*}$, segata da questo píno generico secondo 72 punti appartenenti ad una quintica $a_{5}$, dovrebbe appartenere ad una superficie del quint'ordine $S_{5}$. Cosa assurda perche la polare di $Z_{\sim}$ rispetto $S_{5}$ doprebbe contenere i punti di $\varphi_{42}^{*}$ che hanno pex proiezioni le 294 easpidi.

Si conclude che la serie segata dally $F_{0}$ ha, al massimo, la dimensione $r-s=179$, e quindi 5 almeno di esse, linearmente indipendenti, contengono la $\varphi_{72}^{*}$.

Osservaziont. - Le superfici $F_{9}$ contenenti la $\varphi_{7_{2}}^{*}$ contituiscono un sistema lineare $\Sigma_{s}$ di dimension $s \geq 4$.

Prima di venire al sroondo teorema essenziale dobbinmo premotore ancora aleuni lemmi.

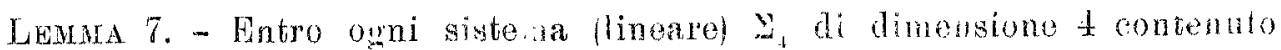
in $\Sigma_{8}$ esiste (almeno) una superficie $F_{9}$ spezzata in an generico piano a (ed in una residaa $F_{8}$ ).

Per dimostrare questo fatto si osservi che la $H_{3}$ passanti $p$ ir la $\varphi_{\gamma z}^{*}$ tawhino sopra un genorico piano a un sistema li curo $C_{9}$ passanti fer 72 punti fissi, appartenenti ad una qualsiasi d tsat, e pertanto su questa, osse segano unit $\hat{y}^{r}$ la eni dimensione si vede encilmente bssare al massimo $r=3$. Segue che in $\Sigma_{4}$ esiste almeno una $F_{9}$ che tontieno if piano $x$.

Inscra 8. - Entro il sistema $\Sigma_{4}$, an generico laseio $\Sigma_{1}$ ili saporfici $H_{n}$ ha una curva base composta della $\varphi_{72}^{*}$ e di una parte residua $\vartheta_{9}^{*}$ (del nono ordine) che non passa per $Z_{\infty}$.

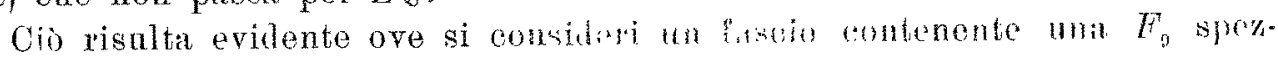
wata in $\alpha$ e in una $E_{8}$. 
Lemma 9. - I coni $H$, osculatori alle varie $F_{9}$ di $\Sigma_{s}$ nel punto quintuplo $Z_{\infty}$, coincidono 0 , almeno, cio accade, per le $F_{9}$ di un sistema (lineare) $\Sigma_{4}$ di dimensione 4 , contenuto in $\Sigma_{s}$.

La cosa sembra evidente, in quanto i suddetti coni $H$, che sono del quinto ordine, devono contenere le 30 rette che dal punto $Z_{\infty}$ proiettano i punti del $T_{30}$ (appartenenti a $\varphi_{12}$ ); ma vi è a temere che i detti coni siano spezzati con una parte in comune, sicchè l'osservazione può costituire una efficace presunzione ma non una dimostrazione.

Questa si ottiene osservando che:

1) Le superficie $\bar{F}_{g}$ aventi in $Z_{\infty}$ la molteplicità 6 , e però del tipo

$$
\bar{F}_{9}=a_{8} z^{3}+a_{7} z^{2}+a_{8} z+a_{8}=0
$$

formano un sistema lineare ${ }^{163}$, e segano sulla $\varphi_{42}^{*}$ gruppi della serie lineare $S^{\prime}=|9 R+3 T|$, la quale ha una certa dimensione $r^{\prime}$.

2) L'indice di specialità della $S^{\prime}$ si calcola osservando che la serie residua di essa rispetto alla serie canonica $C$ :

$$
\left|C-S^{\prime}\right|=|4 R+T|
$$

è segata sulla $\varphi_{42}$ dalle $\Psi_{30}$ per $3 T$ che si scrivono (lemma 4) nella forma

$$
x_{8} a_{27}+x_{4} p_{25}
$$

e perciò ha la dimensione 24 , sicchè

$$
i=25, \quad r^{\prime}=9.42+3.30-334+2 \check{\mathbf{b}}=159 .
$$

3) Segue l'esistenza almeno di un sistema $\infty^{3}$ di $\bar{F}_{9}$ contenenti la $\varphi_{42}^{*}$, e quindi -- ampliando questo mediante una generica $F_{9}$ del sistema $\Sigma_{s}$ l'esistonza del sistema $\Sigma_{4}$ di $F_{8}$ col cono osenlalore $H$ fisso.

Lemas 10. - I 30 punti del $T_{30}$ sono le intersezioni di una quintica $a_{3}=0$ e di una sestica $b_{6}=0$ non aventi parti in comune.

Si considerino infatti due $F_{9}$, una generica nel sistema $\Sigma_{4}$ e l'altra appartenente al sistema $\Sigma_{3}$, rispettivamente di equazione

$$
\begin{array}{r}
a_{5} z^{4}+a_{6} z^{3}+a_{7} z^{2}+a_{8} z+a_{9}=0 \\
b_{6} z^{3}+b_{7} z^{2}+b_{8} z+b_{9}=0 .
\end{array}
$$

I punti $T$ risultano comuni alle curve (quintica e sestica)

$$
a_{5}=0 \text { e } b_{6}=0 \text {. }
$$

D'altra parte queste due curve non possono avere parti in comune, giacchè allora avrebbero una parte in comune $\mathrm{i}$ coni oseulatori, alle due $F_{9}$ sopra scritte, nel punto $Z_{\infty}$ a questo avrebbe allora per la $\varphi_{7 z}^{*}$ molteplicità superiore a' 30 . 
Siamo ora in grado di dimostrare come secondo teorema essenziale il

Teonema 11. - Le superfici $\Phi_{8}$ polari. rispetto alle $F_{9}$ del sistema $\Sigma_{4}$, del loro punto quintuplo $Z_{\infty}$, segano sulla $\varphi_{\tau 2}^{*}$ (come intersezioni variabili) gruppi della $g_{i 2}^{3}=|R+T|$.

Per dimostrare il teorema osserviamo anzitutto che queste polari passano per i punti $K^{*}$ ohe hanno come proiezioni le cuspidi di $\varphi_{42}$, i quali formano un gruppo equivalente, per ipotesi. a $7 R$. Inoltre per una qualunque delle nostre $F_{9}$ contenenti la $\varphi_{72}^{*}$ si consideri la equazione

$$
F_{9}=x_{5} z^{4}+x_{8} z^{3}+x_{7} z^{3}+x_{8} z+x_{9}=0:
$$

accade che la quintica $x_{5}=0$ contenga il gruppo dei punti $T$ (che sono le proiezioni dei punti infinitamente vicini a $Z_{\sim}$ sui vari rami di $\varphi_{72}^{*}$ che passano per $\left.Z_{\infty}\right)$; quindi la relativa polare

$$
\Phi_{\mathrm{s}}=\frac{\partial F_{9}}{\partial z}=\ldots 4 x_{\mathrm{g}} z^{3}+\ldots=0
$$

ha come quintuplo il punto improprio dell' asse $z$ ed è quivi tangente a ciascuno dei $30 \mathrm{rami}$ di $\varphi^{*}$.

Per questi due fatti succede intanto che le $\Phi_{8}$ segano gruppi della serie

$$
|8 R+3 T|-7 R-T=|R+2 T| \text {. }
$$

Per ginngere al nostro assunto occorre riconoscere che la serie $|R+2 T|$ contiene come fisso il gruppo $T$ e quindi, come parti variabili, si riduce alla $|R+T|$.

Infatti, se sommando alla $|R+T|$ il grappo $T$ questa serie si ampliasse, per il lemma di riduzione il gruppo dei 30 punti $T$ dovrebbe imporre meno di 30 condizioni alle aggiunte $\psi_{39}$ che contengono un gruppo della $|R+T|$ e quindi il gruppo $T$ dovrebbe imporre meno di 30 condizioni alle $\psi_{38}$ per $T$.

Ma le $\psi_{38}$ per $T$ segano (su $\varphi_{4 \pm}$ ) la serie $12 R+3 T \mid$ oui appartengono i gruppi segati (su $\varphi_{72}^{*}$ ) dalle superfici $F_{12}$ di equazioni

$$
F_{12}=x_{9} z^{3}+x_{1 z^{2}} z^{2}+x_{11} z+x_{12}=0
$$

(qui, al solito, $x$, indica un polinomio variabile d'ordine $i$ nelle due variabili $x$ e $y$ ).

D'altra parte perche $i$ gruppi segati da queste $B_{12}^{\prime}$ vengano a contenere (ulteriormente) il grappo $T$ occorre che la curva $x_{9}=0$ contenga il gruppo $T$. Ora le $x_{9}$ passanti per il $T$ sono $\infty^{* 4}$ in quauto si scrivono nella forma

$$
x_{9}=x_{4} a_{5}+x_{3} b_{6}
$$

cioè i punti del $T$ impongono esattamente 30 condizioni alle $x_{0}$ e quindi alle $F_{12}$ e (a maggior ragione) alle $\psi_{33}$ gia passanti per il $T_{30}$. 


\section{Conclusione.}

Tutta l'analisi fino ad ora svolta può essere conclusa col seguente

Teorema 12. - La curva $\varphi_{72}^{*}$ è l'intersezione completa di una superficie

$$
H_{9}=a_{5} z^{4}+a_{8} z^{3}+a_{7} z^{2}+a_{8} z+a_{3}=0
$$

(dove ciascuma $a_{i}$ indica un polinomio in $x$ s $y$ d'ordine $i$ ) e della relativa polare

$$
\Phi_{8}=\frac{\partial F_{9}}{\partial z}=4 a_{5} z^{3}+3 a_{6} z^{2}+2 a_{7} z+a_{8}=0 .
$$

Osserviamo infatti che nessuna delle $F_{9}$ del sistema $\Sigma_{4}$ può essere un cono col vertice in $Z_{\infty}$. quindi nessuna delle relative polari (rispetto al polo $\left.Z_{\infty}\right)$ può essere identicamente nulla. Le polari costituiscono quindi un sistema $\Sigma^{\prime}$ di dimensione 4 e poichè taglinno su $\varphi_{72}^{*}$ gruppi della serie $|R+T|$ che ba la dimensione 3 , una di queste polari, e sia essa la polare della $F_{9}$ indicata nell' enunciato, deve contenere la $\varphi_{72}^{*}$.

Osservazione. - Quantunque quasi inutile, si può osservare esplicitamente che il teorema 12 esprime appunto che la $\varphi_{42}$, proiezione della $\varphi_{72}^{*}$, è curva di diramazione del piano quadruplo definito dalla $F_{9}$,

A complemento di tutta la trattazione enunciamo e dimostriamo l'unicità del piano quadruplo diramato dalla $\varphi_{42}$ :

Tronema 13. - Tutti i piani multipli diramati dalla $\varphi_{\$ 2}$ (per i quali le singolarita della $\varphi_{42}$ sono essenziali) sono birazionalmente identici.

Infatti, dal teorema precedente risulta $(7)$ che la $f_{42}$ ammette come forma limite una curva spezzita nelle curve $a_{6}, a_{7}, a_{8}$, ciascuna contata due volte, in modo che ciascun punto comune ad $a_{6}$ ed $a_{8}$ sia limite di quattro nodi e ogni punto comune ad $a_{7}$ con $a_{6}$ e con $a_{8}$ sia limite di tre cuspidi, Segue che alle componenti $a_{6}$ ed $a_{8}$ devono essere collegati scambi del tipo $(1,2)$ e $(3,5)$, mentre alla $a_{\gamma}$ deve essere collegato uno scambio del tipo $(2,3)$. E questo significa l'unicità del piano multiplo.

Osservaztone. - Il ragionamento precedente puo prestarsi a qualche dubbio critico. Ė infatti bensi evidente che le curve doppie $a_{6}$ ed $a_{8}$ contengono di necessità qualche punto di diramazione che ne collega le due parti, onde ad esse compete un unico scambio; ma i 98 punti di diramazione della $a_{7}$ doppia (ciò̀ di collegamento fra le due parti di essa) potrebbero andare tutti a confluire nelle cuspidi. Qui tuttavia basta osservare che la degenerazione della $\varphi_{42}$ si ottiene annullando successivamente i polinomi $a_{5}, a_{6}, a_{7}$ (ciò che porta lo staccamento delle $a_{6}, a_{7}, a_{3}$ contate due volte): dopo lo staccamento di $a_{5}$ (contata due voitel appare chiaro ohe le curve doppie $a_{7}$ ed $a_{8}$ hanno ancora dei punti di diramazione che collegano le loro parti doppie. Pertanto anche alla $a_{\tau}$ doppi: compete un unico seambio.

(7) Cfr. Chisini-Manara, Seconda delle note citate in $(\%)$, 